\title{
Impact of Portland Cement Content on Alkali Activated Bottom Ash
}

\author{
Luís Tambara Júnior, Malik Cheriaf and Janaíde C. Rocha
}

\author{
Laboratory of Waste Valorization and Sustainable Materials (ValoRes), Department of Civil \\ Engineering, PPGEC, Federal University of Santa Catarina (USFC), Campus Trindade, 88040-900 \\ Florianópolis, Brazil, luistambara@gmail.com,malik.cheriaf@gmail.com, janaide.rocha@ufsc.br
}

\begin{abstract}
This study explores the behavior of blended mortars of low reactive bottom ashes (BA) and ordinary Portland cement (OPC) in an alkaline solution. Mortar mixtures incorporating OPC with different replacement levels (0\% to $30 \%$ mass) were studied. Isothermal conduction calorimetric analysis was studied and 28-and 60-day mechanical strength values were found. SEM images was used to identifies porosity structures at 28-day. Water absorption was also investigated. The results revealed different behaviors to low (OPC 2.5 and OPC 5) and moderated (OPC 10 and OPC 30) OPC content. Increase in percentage of Portland accelerated hydration kinetics. There is a second peak formation for moderated OPC content, associated with $C-A-S-H$ gel formation. The partial replacement of bottom ash by OPC tend to reduce the absorption. There is a slower initial water absorption to low OPC content. This behavior is due the higher unreacted BA content, that works as a filler. Otherwise, the increase of water absorption for OPC content is due to the coexistence of Portland cement hydrates and alkali activated reactions.
\end{abstract}

Keywords: Blended Mortars, Alkali Activation, Porosity, Bottom Ash.

\section{Introduction}

Bottom ash (BA) represents $40 \%$ of ashes from the coal-fired thermoelectric power plants (Cheriaf et al., 1999), it is estimated 4.5 million tons of BA per year in Brazil (IEA, 2016). Unlike fly ash, the disposal of bottom ashes goes to settling basin. Studies have shown the use of bottom ashes as an alternative aluminosilicate source for alkali activations (Topçu et al., 2014, Tambara Júnior et al., 2018). However, its performance is worse than fly ash. This is due the less amorphous content, higher unburned coal and low reactive $\mathrm{SiO}_{2}$ and $\mathrm{Al}_{2} \mathrm{O}_{3}$.

An alternative to improving alkali activation BA properties is to increase surface area (through grinding) and to reduce unburnt material, through calcination (Sathonsaowaphak et $a l .$, 2009). Currently in the literature hybrid or blended cements are used to obtain better responses of the material (Garcia-Lodeiro et al., 2016).

Durability remains a property requiring further studies on alkali activations. A problem reported about alkali activation materials is the higher sorptivity when compared with Portland cement. Some works (Collins and Sanjayan, 2000) affirm that this is due the large proportion of fine pores which results in micro-cracks in the matrix, increasing the water capillarity. To reduce the water sorptivity it is necessary add some material that densifies the sample (Rostami and Behgarnia, 2017).

In this article was investigate the influence of ordinary Portland cement (OPC) on the mechanical strength, kinetic of water absorption and pore structure (scanning electron microscope) of blended alkali activation mortar of BA/OPC. The reaction was monitored with isothermal conduction calorimetry to evaluate the heat flow variations in the pastes in the early time of reaction. The OPC was blended from $0 \%, 2.5 \%, 5 \%, 10 \%$ and $30 \%$. 


\section{Experimental Procedure}

\subsection{Materials Characterization}

A Mastersizer 2000 laser granulometer was used to obtain particle size distribution of OPC and BA (Figure 1) and the powder was dispersed in isopropanol. It is seen that the BA used in this study contained a finer particle size than BA. Where $86 \%$ of OPC and $99.9 \%$ of BA particle size measured under $45 \mu \mathrm{m}$, in agreement with the size particle parameter used to improve ash reactivity (Fernández-Jiménez e Palomo 2003).

The BA and OPC oxide analysis and clinker phase compositions are presented in Table 1. To the cement the phase was estimated based on Bogue calculation method. To BA was performed a Rietveld refinement method (Rietveld, 1969) using X-ray diffraction test data. To determine the amorphous and crystalline content in the BA sample, the analytical reagent corundum $\left(\mathrm{Al}_{2} \mathrm{O}_{3}\right)$ was added in a total of $10 \%$ by mass as an internal standard. The OPC is a type III and the BA is classified as ash type F (low calcium content).

The sand used in the mortar mixtures was a natural fine sand. It was obtained a relative specific density of $2600 \mathrm{~kg} / \mathrm{m}^{3}$, water absorption of $1.6 \%$, maximum grain size of $1.2 \mathrm{~mm}$ and minimum grain size of $0.15 \mathrm{~mm}$. The sand showed a fineness modulus of 1.83 .

Table 1. Chemical composition.



A combination of sodium hydroxide $(\mathrm{NaOH})$ pellets, water distilled and sodium silicate solution $\left(\mathrm{Na}_{2} \mathrm{SiO}_{3}\right)$ was used as an alkaline activator $\left(\mathrm{SiO}_{2}=26.5 \% ; \mathrm{Na}_{2} \mathrm{O}=10.6 \% ; \mathrm{H}_{2} \mathrm{O}=\right.$ $62.9 \%$; and density of $1.39 \mathrm{~g} / \mathrm{cm}^{3}$ ). Initially a $14 \mathrm{M} \mathrm{NaOH}$ solution was prepared with distilled water, waiting up to room temperature. After that a ratio of $1: 2 \mathrm{Na}_{2} \mathrm{SiO}_{3}$ to $\mathrm{NaOH}$ solution were prepared. A superplasticizer additive based on polycarboxylate ether, with solids content of 0.22 , was used to adjust the workability (flow value $>250 \mathrm{~mm}$ ) required for self-leveling mortars.

To evaluate the effect of OPC content on the microstructure of blended alkali-activated mortars, it was prepared 5 mixtures. The sum of OPC and BA was considerate as binder. Hydrated mortars were prepared using the same liquid-to-binder $(1 / b)$ ratio of 0.55 . The substitution in mass of BA for Portland cement was: $0 \%$ (i.e., only bottom ash), $2.5 \%, 5 \%, 10 \%$ and $30 \%$. The binder and sand ratio were kept constant from 1:2 (in mass). The molar ratios were between 2.6-3.1 to $\mathrm{Si} / \mathrm{Al}, 0.27-0.30$ to $\mathrm{Na}_{2} \mathrm{O} / \mathrm{SiO}_{2}$ and equal to 12.3 to $\mathrm{H}_{2} \mathrm{O} / \mathrm{Na}_{2} \mathrm{O}$. 


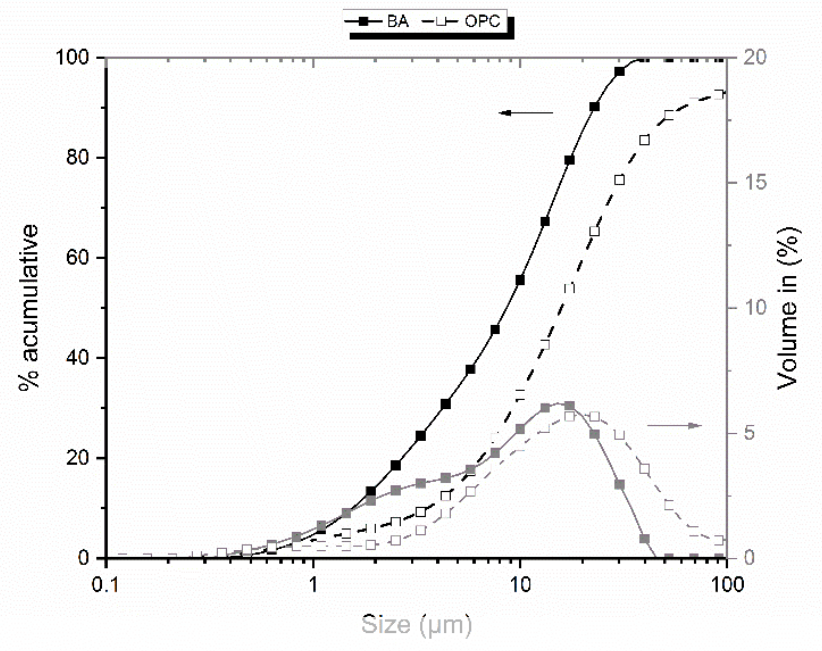

Figure 1. Particle size of OPC and BA.

\subsection{Experimental Procedure}

The mixing method consisted of an initial premix of the solutions $\left(\mathrm{NaOH}\right.$ and $\left.\mathrm{Na}_{2} \mathrm{SiO}_{3}\right)$ until room temperature. After that, a dry mix powder of cement, bottom ash and sand of each

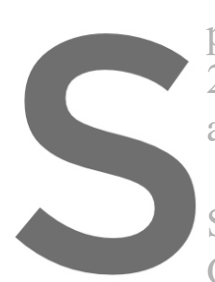
proportion was executed. The solid/liquid mixing was performed in a planetary mixer, the first 2 minutes at $150 \mathrm{rpm}$ and one last mimute at $300 \mathrm{rpm}$. Then the mortars were added to th and cured for $24 \mathrm{~h}$ at $80^{\circ}$

Compressive streng Standard NBR 13279 Calorimetric tests we conduction isotherm calorimeter TAM Air-Thermometric. The pastes were manually mixed for

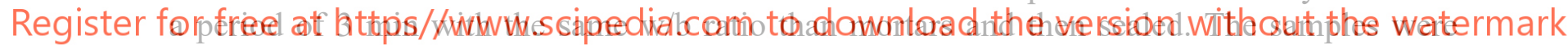
immediately placed in the calorimeter, under the same conditions as the mortars $\left(80^{\circ} \pm 1^{\circ} \mathrm{C}\right)$.

To stop the reaction at the test ages for microstructural analysis, the samples were kept in an oven at $40^{\circ} \mathrm{C}$ until constancy of mass. Water absorption was evaluated over time for 24 hours through a method of Mariotte's bottle developed of laboratory ValoRes-UFSC. To analyze the microstructure images, the samples were carbon-coated and mounted on electron microscope slides for study under a JEOL JSM-6390SL (15.0kV) with energy-dispersive X-ray spectroscopy (EDS) performed at Central Laboratory of Electron Microscopy (LCME-UFSC).

\section{Results and Discussion}

\subsection{Isothermal Calorimetry and Compressive Strength}

The effect of OPC content on the rate of heat release and total heat release during alkali activation of the BA is shown in Figure 2. For all the samples is seen a faster reaction at $80^{\circ} \mathrm{C}$, due to this it was not possible to detect the first peak associated aluminosilicate dissolution, as observed in literature (Palomo et al., 1999; Garcia-Lodeiro et al., 2013). For the alkali 
activation only with BA (OPC0) it is seen the lowest heat release and total heat. This is due its insufficient dissolution phases that start the formation of N-A-S-H gel.

Two different behaviors were observed according to the OPC content on the hybrid cements. Results are presented as low OPC content (OPC2.5 and OPC5) and moderate OPC content (OPC10 and OPC30). Even in small quantities, increasing OPC content accelerates the activation reaction. To low OPC content (Figure $2 \mathrm{a}$ ) it is noticed the formation of a single peak in heat evolution. The increment of OPC content resulted in a higher heat release and total heat, this indicates an increase of reaction degree. OPC2.5 and OPC5 showed a total heat at $6 \mathrm{~h}$ of $59.79 \mathrm{~J} / \mathrm{g}$ and $77.73 \mathrm{~J} / \mathrm{g}$, respectively.

Figure $2 \mathrm{~b}$ shows heat flow curve and total heat for the alkali activation with moderate OPC content. It is observed a more intense and rapid first peak compared with low OPC content, followed by a short dormant period (occurs up to 15 minutes of activation). OPC10 and OPC 30 presented a total heat at $6 \mathrm{~h}$ of $148.48 \mathrm{~J} / \mathrm{g}$ and $126.76 \mathrm{~J} / \mathrm{g}$, respectively.

The quick first peak formation may be associated with an accelerated Si and Al dissolution and N-A-S-H formation. Also, a secondary peak starts after 15 minutes of activation. After the first gel formation the $\mathrm{pH}$ of pore solution decreases by which the calcium provided from OPC begins to react and form a (N,C)-A-S-H gel type (Garcia-Lodeiro et al., 2016).

OPC30 showed the highest total heat up to $1 \mathrm{~h}$ of activation, however, OPC10 overcame the total heat in higher period. Martinez-Ramirez and Palomo (2001) showed that OPC hydration is retarded when alkali concentrations increases. This suggests that is greater amount of inner


anhydrous cement to OPC30 than OPC10.

Register for
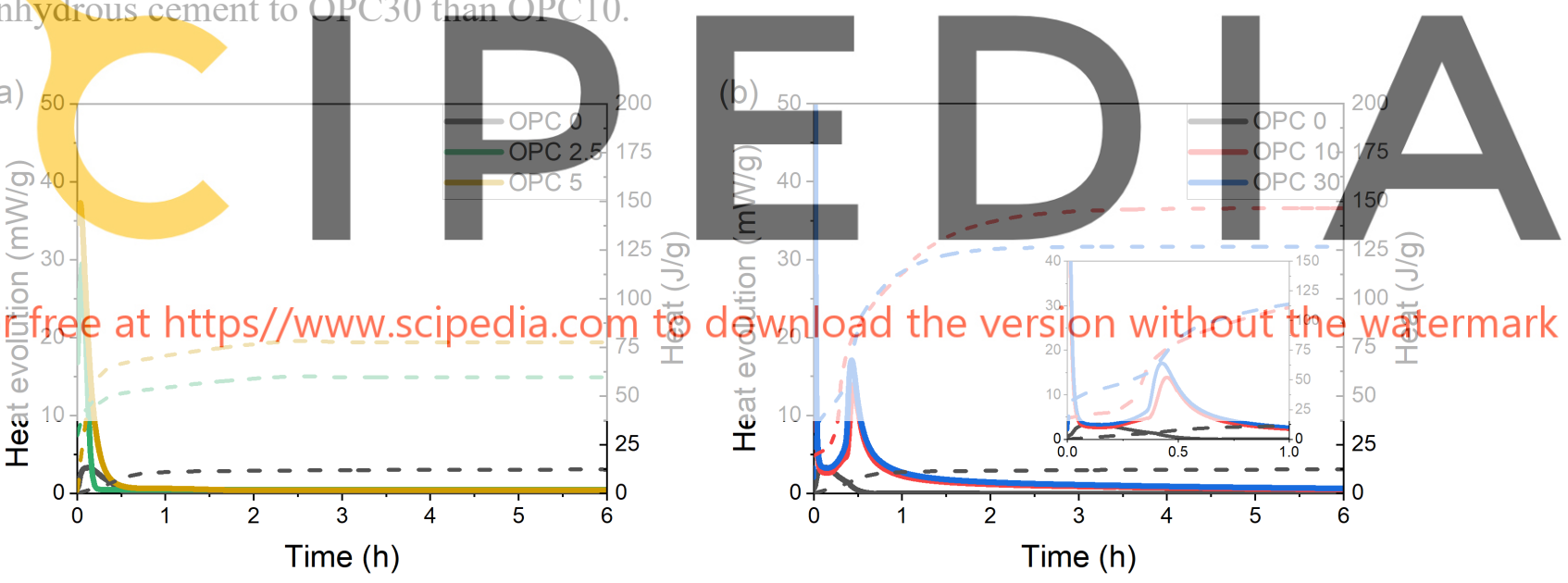

Figure 2. Heat flow and cumulative heat from the low OPC content (a) and moderate OPC content (b) alkali

Activation.

Compressive strength was evaluated to later ages of $28 \mathrm{~d}$ and 60 days (Figure 3). OPC0 showed no strength development over the time of 60 days. This result shows that the exclusive alkali activation of BA results in a very slow evolution of reaction. The gain of compressive strength from $28 \mathrm{~d}$ to $60 \mathrm{~d}$ of OPC2.5, OPC5 correspond to $8.8 \%, 12.8 \%$ to low OPC content, a result in agreement with heat flow calorimetric analysis. To moderate OPC content the compressive strength for $28 \mathrm{~d}$ and $60 \mathrm{~d}$ increases $19.9 \%$ and $11.6 \%$ to OPC10 and OPC 30 , respectively. There is observed an optimum OPC content of $10 \%$ to blended mortar, increasing the bottom ash reaction. 


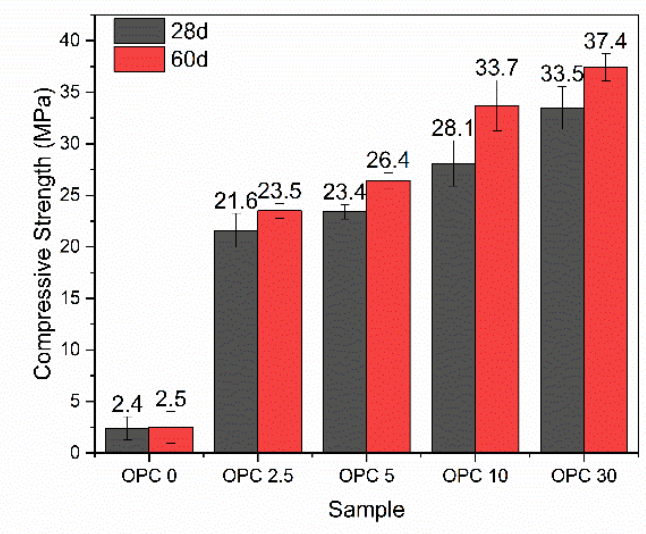

Figure 3. Compressive strength to $28 \mathrm{~d}$ and $60 \mathrm{~d}$.

\subsection{Water Absorption}

Water transportation of mortars is an important property that mainly influences its durability. Figure 4 shows water absorption over a $24 \mathrm{~h}$ of experiment to low and moderate OPC mortars at $1 \mathrm{~d}$ and $28 \mathrm{~d}$. The water absorption of the blended mortars reported in this work were obtained in the final rage of $2.27 \%-10.41 \%$ and $1.57 \%+3.30 \%$ for $1 \mathrm{~d}$ and $28 \mathrm{~d}$ respectivery At $1 \mathrm{~d}$. hydration, it is observed higher absorption of activation with increased OrC dostge.

At 28 d was observed to all samples a reduction of water absorption. Compared w hydration, OPC30, OPC10, OPC5 and OPC2.5 presented a water absorption reduction of $57.04 \%, 81.17 \%, 68.59 \%$ and $30.84 \%$, respectively. Water absorption reduction is moderate OPC mortars. It was observed that OPC10 showed the lowest water absorption to 28 days. The authors associate this behavior to the pore filling due best compatibility of last


2018).
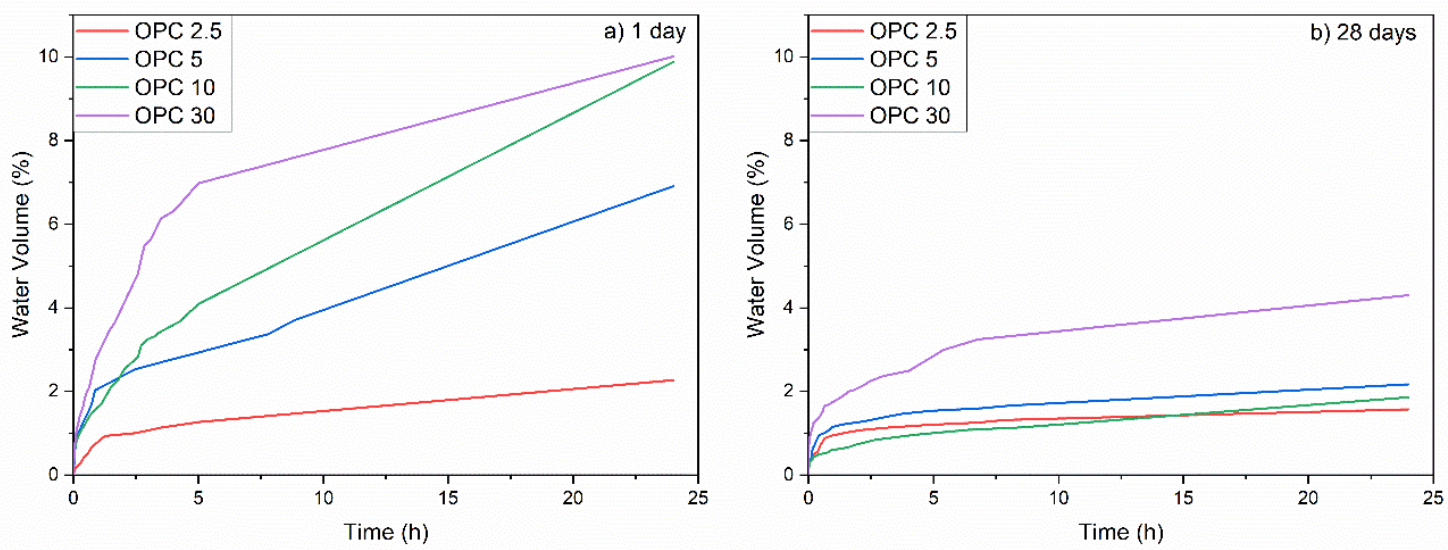

Figure 4. Water absorption of low OPC moderate OPC content at 1 day (a) and 28 days (b) of reaction. 


\subsection{SEM}

As Figure 5a shows, the bottom ash after treatment presented predominance of irregular particles with few spherical particles, which difficult the activation. Figure $5 \mathrm{~b}$ shows the OPC0 at 28 days of activation, where there is a larger presence of unreacted BA and insufficient gel formation, featuring in a higher porosity and low compressive strength (Figure 3).

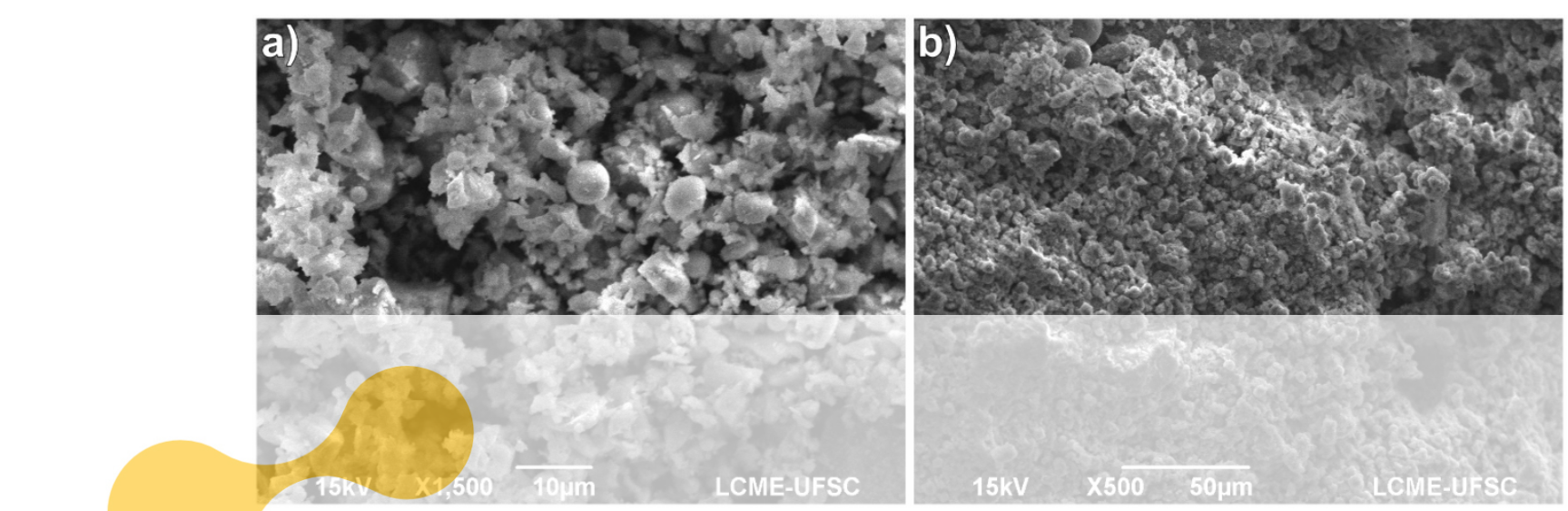

Figure 5. SEM images of a) BA and b) OPC0 hydrated at 28 days.



mortars. As calorimetry analysis shows, the improve of OPC content results in a higher energy

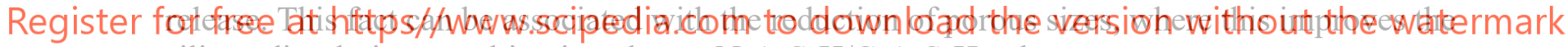
silicon dissolution, resulting in a denser $\mathrm{N}-\mathrm{A}-\mathrm{S}-\mathrm{H} / \mathrm{C}-\mathrm{A}-\mathrm{S}-\mathrm{H}$ gels.

Moderate OPC mortars resulted in a reduced presence of the larger porous size. OPC30 and OPC10 presented similar pore size range of $20 \mu \mathrm{m}-140 \mu \mathrm{m}$. Otherwise, it was observed the lower water absorption to the OPC10, that can be associated to a higher microporosity to OPC 30 mortar. 

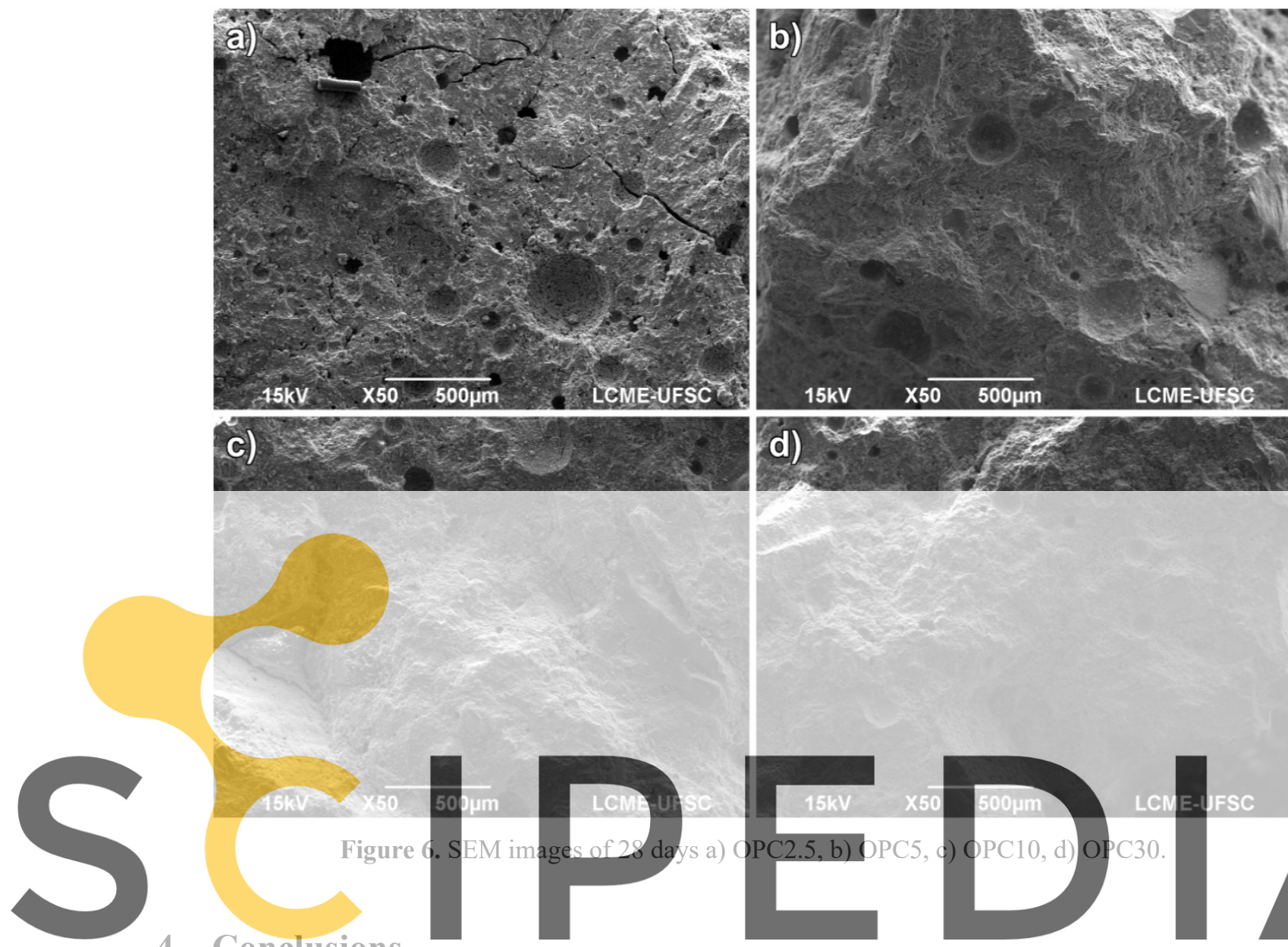

\section{Conclusions}


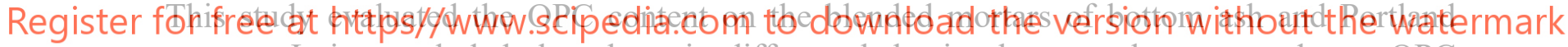
cement. It is concluded that there is different behavior between low to moderate OPC replacement, but even in low content (2.5\% and 5\%) there is a good contribution to activate bottom ashes with low reactivity.

To all the samples, as the OPC replacement increased the mechanical strength and the water absorption increases. This improved performance may be due to a denser microstructure generated to C-A-S-H gel formation observed through microstructural and calorimetry analysis that showed a secondary peak to moderate OPC content.

Furthermore, the $10 \%$ substitution of OPC significantly improved the parameters of durability, associated with the higher strength development at later ages and lower water absorption, due to its suitable $\mathrm{Ca}^{+}$content provided by OPC. The increase of compressive strength and water absorption of OPC 30 may be associated with a reduction of macroporosity but higher microporosity.

\section{Acknowledgements}

This study was sponsored by the Coordenação de Aperfeiçoamento de Pessoal de Nível Superior (CAPES), Conselho Nacional de Desenvolvimento Científico e Tecnológico (CNPq). We are grateful CNPq, CAPES and LCME-UFSC for assistance in scanning electron microscopy operation. 


\section{ORCID}

L. Tambara Júnior: https://orcid.org/0000-0002-3269-4911

Malik Cheriaf: https://orcid.org/0000-0001-5440-2636

Janaíde C. Rocha: https://orcid.org/0000-0003-1074-3230

\section{References}

ABNT (2005). NBR 13279: Mortars applied on walls and ceilings - Determination of the flexural an the compressive strength in the hardened stage. (In Portuguese).

Cheriaf, M., Cavalcante Rocha, J. and Péra, J. (1999). Pozzolanic properties of pulverized coal combustion bottom ash, Cem. Concr. Res. 29 1387-1391. doi:0008-8846/99/\$.

Collins F. and Sanjayan G.J. (2000). Effect of pore size distribution on drying shrinkage of alkali-activated slag concrete, Cem Concr Res 30 1401-1406.

Fernández-Jiménez and A. Palomo, A. (2003). Characterisation of fly ashes. Potential reactivity as alkaline cements. Fuel, 82(18), 2259-2265. doi: 10.1016/S0016-2361(03)00194-7

Garcia-Lodeiro, I. Fernandez-Jimenez and A. Palomo, A. (2013). Hydration kinetics in hybrid binders: Early reaction stages. Cement and Concrete Composites. 39, 82-92. doi: 10.1016/j.cemconcomp.2013.03.025

Garcia-Lodeiro, I., Donatello, S., Fernández-Jiménez, A. and Palomo, A. (2016). Hydration of hybrid alkaline cement containing a very large proportion of fly ash: A descriptive model, Materials. 9 doi:10.3390/MA9070605

IEA, International Energy Agency Coal Information 2015 with 2015 data. (2016). Energy Technol. Perspect, https://www.oecd-ilibrary.org/energy/coal-information-2016 coal-2016-en, Accessed date: 14 October 2019

Martínez-Ramírez, S. and Palomo, A. (2001). OPC hydration with highly alkaline solutions. Advances in Cement Research 13(3) 123-129.

Palomo, A. Grutzeck, M.W and Blanco MT (1999) Alkali-activated fly ashes A cement for the future, Cem


Concr. Res. 29 1323-1329.

Rietveld, H. M. (1969). Crystallography, 2(2),

Rostami, M. and Behfarnia, Construction and Build

Sathonsaowaphak, A., Chi

geopolymer mortar. Journal of Hazardous Materials 168 (1) 44-50. doi: 10.1016/j.jhazmat.2009.01.120.

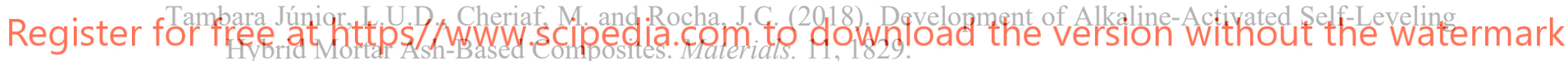

Topçu, I.B., Toprak, M.U. and Uygunoğlu, T. (2014). Durability and microstructure characteristics of alkali activated coal bottom ash geopolymer cement, J. Clean. Prod. 81 211-217. doi:10.1016/j.jclepro.2014.06.037 